\title{
Electrodeposition of Sm-Co Alloy Films with Nanocrystalline/Amorphous Structures from a Sulphamate Aqueous Solution
}

\author{
Minling Xie, Liqun Zhu, Weiping Li, Huicong Liu* ${ }^{*}$ Tianli Zhang ${ }^{*}$ \\ Key Laboratory of Aerospace Materials and Performance (Ministry of Education), School of Materials \\ Science and Engineering, Beihang University, No.37 Xueyuan Road, Haidian District, Beijing 100191, \\ China \\ *E-mail: liuhc@ buaa.edu.cn, tlzhang@buaa.edu.cnz
}

doi: $10.20964 / 2017.12 .07$

Received: 15 July 2017 / Accepted: 22 September 2017 / Published: 12 November 2017

Sm-Co alloys as permanent magnetic materials have attracted considerable interest for their application in the miniaturization of electronic devices. However, a facile method for depositing SmCo alloy films is still urgently needed. In this work, we exploited a simple aqueous electrodeposition approach to prepare Sm-Co films. Electrodeposition was realized by galvanostatic electrolysis from a stable sulfamate aqueous solution. The influence of the applied current density on the composition, morphology, structure and magnetic properties of the Sm-Co films was studied. Samarium was successfully incorporated into the films by the induced effect of $\mathrm{Co}$ (II) ions. Manipulating the current density varied the samarium content from 5.8 to 25.3 at $\%$. Sm-Co nanosheets were discovered and were determined to have a mixed nanocrystalline/amorphous character. Furthermore, the electrodeposited Sm-Co films exhibited an obviously enhanced coercivity compared with that of other reported films.

Keywords: Sm-Co films; Electrodeposition; Aqueous solution; Nanosheets;

Nanocrystalline/amorphous

\section{$\underline{\text { FULL TEXT }}$}

(C) 2017 The Authors. Published by ESG (www.electrochemsci.org). This article is an open access article distributed under the terms and conditions of the Creative Commons Attribution license (http://creativecommons.org/licenses/by/4.0/). 